\title{
Threshold and Stability Results of a New Mathematical Model for Infectious Diseases Having Effective Preventive Vaccine
}

\author{
Sümeyye Çakan ${ }^{1}$ \\ ${ }^{1}$ Department of Mathematics, Faculty of Science and Arts, İnönü University, Malatya, Turkey
}

\author{
Article Info \\ Keywords: Mathematical Modelling, \\ Local Asymptotic Stability, Global At- \\ tractivity, Global Asymptotic Stability, \\ Vaccine Effect, Disease-free Equilib- \\ rium Point, Basic Reproduction Num- \\ ber. \\ 2010 AMS: 34D05, 34D20, 34D23, \\ 34K20, 92B05, 92D25, $92 D 30$. \\ Received: 21 Feb 2021 \\ Accepted: 19 August 2021 \\ Available online: 31 August 2021
}

\begin{abstract}
This paper evaluates the impact of an effective preventive vaccine on the control of some infectious diseases by using a new deterministic mathematical model. The model is based on the fact that the immunity acquired by a fully effective vaccination is permanent. Threshold $\mathscr{R}_{0}$, defined as the basic reproduction number, is critical indicator in the extinction or spread of any disease in any population, and so it has a very important role for the course of the disease that caused to an epidemic. In epidemic models, it is expected that the disease becomes extinct in the population if $\mathscr{R}_{0}<1$. In addition, when $\mathscr{R}_{0}<1$ it is expected that the disease-free equilibrium point of the model, and so the model, is stable in the sense of local and global. In this context, the threshold value $\mathscr{R}_{0}$ regarding the model is obtained. The local asymptotic stability of the disease-free equilibrium is examined with analyzing the corresponding characteristic equation. Then, by proved the global attractivity of disease-free equilibrium, it is shown that this equilibria is globally asymptotically stable.
\end{abstract}

\section{Introduction}

It has been seen that epidemics have had major effects and leave deep remains on human lives throughout history. To prevent and control the spread of epidemic, the examination of its' dynamics has an important role. In this context, mathematical modelling in epidemiology provides understanding and explanation of the underlying mechanisms that influenced the spread of disease, and it suggests control strategies. The COVID-19 pandemic, which emerged at the end of 2019 and is the most devastating epidemic of recent times, has seriously shaken humanity as a global threat. Modeling and analysis studies in mathematical epidemiology have focused on this ground in conjunction with this compelling and exhausting epidemic and many authors have made various contributions to this field with the help of the models they have constituted. [1-3] and references therein are among some current studies on this subject.

While dealing with mathematical modeling spread of disease, in order to formulate the transmissions of an epidemic, the population in a region is often divided into different compartments, and the models, which formulate the relations between these compartments, are called as compartmental models. In the literature, there are many compartmental models provided basic principles for the spread of a disease in a population. Kermack and McKendrick with their study [4] have pioneered in studies using compartmental mathematical models. In the model proposed by Kermack and McKendrick in 1927, the population was divided into three compartments: a susceptible compartment labelled $S$, in which all individuals are susceptible to the disease; an infected compartment labelled $I$, in which all individuals are infected by the disease and have infectivity; and a removed compartment labelled $R$, in which there exist the individuals consist whose infectiousness finished. This model is called as "SIR model" based on the initials of the group names.

Then, a lot of authors have tackled with various details to carry further forward this model. Adding a vaccination compartment is just one of these details. Immunization with vaccines is among the most effective methods of protection from infectious diseases which are common in the society and which have high contamination properties. Until today, many studies including the epidemic models with vaccination have been introduced. The references [5] and [6] are just two of them.

In mathematical epidemiology, the course of the disease in the population associates with whether the basic reproduction number is greater than 1 , or not. It can be made the comments "If $\mathscr{R}_{0}>1$, there is an increase in the speed of the spread; if $\mathscr{R}_{0}<1$, there is a decrease in the epidemic rate and the epidemic is under control; if $\mathscr{R}_{0}=1$, speed of the spread is constant". The value $\mathscr{R}_{0}$ is very important since it can tell us whether the population is at risk about the disease. Therefore, calculating this value for any disease in any population is unvaluable. 
Considering the unfavorable conditions brought on by an epidemic disease in a population, studies to reduce the spread of the disease have, of course, great significance. In order that the disease dies out in the population, it needs that $\mathscr{R}_{0}<1$ and additionally the disease-free equilibrium point of the projected model is stable when $\mathscr{R}_{0}<1$. In other words, the effort required to prevent an outbreak or to eliminate an infection in a population should be directed towards ensuring that the value $\mathscr{R}_{0}$ is less than 1 . In addition, it is expected that the disease-free equilibrium point of the model and so the model is stable in the sense of locally and globally, when $\mathscr{R}_{0}<1$.

Hence, for the past decades, many studies have been proposed on local and global stabilities of the disease-free equilibria, [7-14].

The aim of this study is to present a model that reflects the fact that the protective effect may not occur immediately after the vaccine is administered and the fact that this protection may not be fully effective even if the protective effect of the vaccine has started. The model uses a system of nonlinear ordinary integro-differential equations with delay to explain this fact. The study focuses on the basic reproduction number of this new compartmental epidemic model and on the local and global stabilities of the disease-free equilibria of model.

\section{Model Description and Assumptions}

In this study in which we aim to propose and analyze a new SVEIR epidemic model with delay, we divide a population $(N)$, among which some individuals are known to be exposed to infectious disease, into five classes shaped like Susceptible $(S)$, Vaccinated $(V)$, Exposed $(E)$, Infectious $(I)$ and Removed $(R)$ individuals.

The transitions between the compartments are expressed by the following system:

$$
\begin{aligned}
\frac{d S}{d t} & =b-\beta_{1} S(t) I(t)-q S(t)-\mu S(t) \\
\frac{d V}{d t} & =q S(t)-q \beta_{2} I(t) \int_{0}^{h} e^{-\mu \tau} S(t-\tau) d \tau-v q \int_{h}^{\infty} e^{-\mu \tau} S(t-\tau) d \tau-(1-v) q \beta_{3} I(t) \int_{h}^{\infty} e^{-\mu \tau} S(t-\tau) d \tau-\mu V(t) \\
\frac{d E}{d t} & =\beta_{1} S(t) I(t)+q \beta_{2} I(t) \int_{0}^{h} e^{-\mu \tau} S(t-\tau) d \tau+(1-v) q \beta_{3} I(t) \int_{h}^{\infty} e^{-\mu \tau} S(t-\tau) d \tau-\gamma E(t)-\eta E(t)-\mu E(t) \\
\frac{d I}{d t} & =\gamma E(t)-\alpha I(t)-\delta I(t)-\mu I(t) \\
\frac{d R}{d t} & =v q \int_{h}^{\infty} e^{-\mu \tau} S(t-\tau) d \tau+\eta E(t)+\alpha I(t)-\mu R(t)
\end{aligned}
$$

Where $S(t), V(t), E(t), I(t)$ and $R(t)$ denote the numbers of susceptible, vaccinated, exposed, infectious, and removed individuals at time $t$, respectively. The total population size at time $t$ is $N(t)$ and $N(t)=S(t)+V(t)+E(t)+I(t)+R(t)$ for all $t \geq 0$. Also all these functions are nonnegative.

All parameters belonging to the model are nonnegative constants and all newborn individuals get involved in the population by entering to the susceptible class with a constant rate $b$.

The effective contact rate between infectious individuals and susceptibles is $\beta_{1}$. Also $\beta_{2}$ is the effective contact rate between infectious and the individuals into the vaccinated group whose vaccinated at time $t-\tau$ and vaccine effect has not yet started. $\beta_{3}$ represents the effective contact rate between infectious and susceptibles whose vaccinated at time $t-\tau$ and vaccine effect has started, but whose their contribution rate to the protection provided by the vaccine less than 1 .

We assume that $q$ is the rate of vaccinated individuals within susceptible group and $\mu$ is natural death rate in each compartment, $\delta$ is death rate derived from pathogen causing to outbreak. $\gamma$ indicates the rate at which exposed individuals become infectious. Also, $\alpha$ and $\eta$ represent the transition rates from compartments consist of infectious and exposed individuals to the compartment $R$, respectively.

Active immunization, the way of activating the body's immune system through vaccination, is often applied before encountering with microorganisms, that is, before encountering contamination, in order to create antibodies / antitoxins against infectious diseases having high contagious properties and being severe consequences.

In immunization via vaccine, it requires a certain time (weeks or months) for antibody/antitoxin formation. In other words, protective effect (antibody/antitoxin) does not occur immediately after the vaccine is administered or after the first dose of the vaccine. We assume that the protective effect of the vaccine begins $h$ time after vaccination. That is, protection of an individual who vaccinated at time $t$, begins at time $t+h$. So the term $q \int_{0}^{h} e^{-\mu \tau} S(t-\tau) d \tau$ indicates the number of individuals who have been vaccinated at time $t-\tau$ and their protection effect of vaccine has not yet started since the time threshold $h$ does not completed, at time $t$. In this period that the vaccine does not yet form any effect, a vaccinated individual who have not yet any protection enters to compartment $E$ if exposing to the infectious agent with a sufficiently effective contact with infectious individuals. This transition is expressed with the term $q \beta_{2} I(t) \int_{0}^{h} e^{-\mu \tau} S(t-\tau) d \tau$ in the model.

It is also another fact that no vaccine has a $100 \%$ protective effect. In some individuals, the body's response to the vaccine may be weak because of various reasons. Therefore, the vaccine protection is lower for such individuals, and it can be seen that the protective efficacy in after vaccination is not fully formed. By considering this situation, the protection rate provided by the vaccine has been shown by $v$ in the model. As a result of effective contact between infectious individuals and the vaccinated (but are susceptible ) individuals whose protection provided by the vaccine is less than 1 , enter to latent compartment by exposing to the infectious agent. This transition is denoted by $(1-v) q \beta_{3} I(t) \int_{h}^{\infty} e^{-\mu \tau} S(t-\tau) d \tau$.

On the other hand the individuals, whose protection level provided by the vaccine is 1 and so have full protection, include to the class $R$ by gaining immunity. This transition is represented by the term $v q \int_{h}^{\infty} e^{-\mu \tau} S(t-\tau) d \tau$. 


\subsection{Qualitative Analysis of the Model}

In this section, we find the feasible positive invariant region, equilibrium points and basic reproduction number belong to the model.

\subsubsection{Feasible Positive Invariant Region}

Theorem 2.1. The set

$$
\Upsilon=\left\{S \in C\left([-\tau, \infty),\left[0, \frac{b}{\mu}\right]\right),(V, E, I, R) \in C\left(\mathbb{R}_{+},\left[0, \frac{b}{\mu}\right]\right): N(t) \leq \frac{b}{\mu}\right\}
$$

is positively invariant and bounded for the model.

Proof. Summing up the five equations of system (2.1), we obtain

$$
\begin{aligned}
N^{\prime}(t) & =\frac{d S}{d t}+\frac{d V}{d t}+\frac{d E}{d t}+\frac{d I}{d t}+\frac{d R}{d t} \\
& =b-\mu(S(t)+V(t)+E(t)+I(t)+R(t))-\delta I(t) \\
& \leq b-\mu N(t) .
\end{aligned}
$$

For the solution of this nonlinear differential inequality, we investigate the differential equation

$$
N^{\prime}(t)+\mu N(t)=b
$$

From solving of this ordinary differential equation we get $N(t)=b / \mu+c e^{-\mu t}$. For the initial condition $t=0$, we find the solution as

$$
N(t)=N(0) e^{-\mu t}+\frac{b}{\mu}\left(1-e^{-\mu t}\right) .
$$

The right side of the equality (2.4) is the maximal solution of (2.3) by Standard Comparison Theorem [15]. Hence we have the inequality

$$
N(t) \leq N(0) e^{-\mu t}+\frac{b}{\mu}\left(1-e^{-\mu t}\right)
$$

for every $t \geq 0$. If $N(0) \leq b / \mu$ then $N(t) \leq b / \mu$ for all $t>0$ and so the set $\Upsilon$ given by (2.2) is positively invariant for the system (2.1). Also, it is clearly seen that $N(t)$ is bounded above with $b / \mu$.

This region can be considered as a feasible bounded region which is enough to study epidemiologically and mathematically.

Since the population $V(t)$ and $R(t)$ do not feature in remainder equations of (2.1), we can study on the following reduced system (2.5):

$$
\begin{aligned}
S^{\prime}(t) & =b-\beta_{1} S(t) I(t)-(q+\mu) S(t), \\
E^{\prime}(t) & =\beta_{1} S(t) I(t)+q \beta_{2} I(t) \int_{0}^{h} e^{-\mu \tau} S(t-\tau) d \tau+(1-v) q \beta_{3} I(t) \int_{h}^{\infty} e^{-\mu \tau} S(t-\tau) d \tau-(\gamma+\eta+\mu) E(t), \\
I^{\prime}(t) & =\gamma E(t)-(\alpha+\delta+\mu) I(t) .
\end{aligned}
$$

\subsubsection{Disease-Free Equilibrium Point}

Now, let us find the disease-free equilibrium point of the model (2.5). To do this we take $S(t)=S_{0}, V(t)=V_{0}, E(t)=E_{0}$ and $I(t)=I_{0}=0$. So, for the system of algebraic equations

$$
\begin{aligned}
0 & =b-\beta_{1} S_{0} I_{0}-(q+\mu) S_{0}, \\
0 & =\beta_{1} S_{0} I_{0}+q \beta_{2} S_{0} I_{0} \int_{0}^{h} e^{-\mu \tau} d \tau+(1-v) q \beta_{3} S_{0} I_{0} \int_{h}^{\infty} e^{-\mu \tau} d \tau-(\gamma+\eta+\mu) E_{0}, \\
0 & =\gamma E_{0}-(\alpha+\delta+\mu) I_{0},
\end{aligned}
$$

the disease-free equilibrium point is obtained as

$$
P_{D F}=\left(S_{0}, E_{0}, I_{0}\right)=\left(\frac{b}{q+\mu}, 0,0\right)
$$




\subsubsection{Basic Reproduction Number}

As is known from mathematical epidemiology, the basic reproduction number $\mathscr{R}_{0}$ is used to measure the transmission potential of a disease. It is the average number of secondary infections produced by a typical case of an infection in a population in which everyone is susceptible, [16].

In the following part, we calculate this critical threshold value $\mathscr{R}_{0}$ for the model with the help of next generation matrix method, [17].

Let us take $X=(E, I, S)^{T}$ and write the system (2.5) in the form

$$
\underbrace{\left[\begin{array}{c}
\dot{E} \\
\dot{I} \\
\dot{S}
\end{array}\right]}_{\frac{d X}{d t}}=\underbrace{\left[\begin{array}{c}
\beta_{1} S(t) I(t)+q \beta_{2} I(t) \int_{0}^{h} e^{-\mu \tau} S(t-\tau) d \tau+(1-v) q \beta_{3} I(t) \int_{h}^{\infty} e^{-\mu \tau} S(t-\tau) d \tau \\
0
\end{array}\right]}_{\mathscr{K}(X)}-\underbrace{\left[\begin{array}{c}
(\gamma+\eta+\mu) E(t) \\
(\alpha+\delta+\mu) I(t)-\gamma E(t) \\
\beta_{1} S(t) I(t)+(q+\mu) S(t)-b
\end{array}\right]}_{\mathscr{V}(X)}
$$

that is

$$
\frac{d X}{d t}=\mathscr{K}(X)-\mathscr{V}(X)
$$

The values at the disease-free equilibrium point $P_{D F}$ of the derivatives of $\mathscr{K}(X)$ and $\mathscr{V}(X)$ with respect to $E, I, S$, respectively, come in sight with the following Jacobian matrices:

$$
J_{\mathscr{K}}\left(P_{D F}\right)=\left[\begin{array}{ccc}
0 & J_{\mathscr{K}}^{12}\left(P_{D F}\right) & J_{\mathscr{K}}^{13}\left(P_{D F}\right) \\
0 & 0 & 0 \\
0 & 0 & 0
\end{array}\right]
$$

such that

$$
J_{\mathscr{K}}^{12}\left(P_{D F}\right)=\beta_{1} S_{0}+q \beta_{2} S_{0} \int_{0}^{h} e^{-\mu \tau} d \tau+(1-v) q \beta_{3} S_{0} \int_{h}^{\infty} e^{-\mu \tau} d \tau
$$

and

$$
J_{\mathscr{K}}^{13}\left(P_{D F}\right)=\beta_{1} I_{0}+q \beta_{2} I_{0} \int_{0}^{h} e^{-\mu \tau} d \tau+(1-v) q \beta_{3} I_{0} \int_{h}^{\infty} e^{-\mu \tau} d \tau
$$

and also

$$
J_{\mathscr{V}}\left(P_{D F}\right)=\left[\begin{array}{ccc}
\gamma+\eta+\mu & 0 & 0 \\
-\gamma & \alpha+\delta+\mu & 0 \\
0 & \beta_{1} S_{0} & \beta_{1} I_{0}+(q+\mu)
\end{array}\right]
$$

Taking into account that infections can only exist in compartments $E$ and $I$, the block matrices $K$ and $V$ are formed as

$$
K=\mathscr{K}_{2 \times 2}=\left[\begin{array}{cc}
0 & K_{12} \\
0 & 0
\end{array}\right]
$$

such that

$$
K_{12}=\beta_{1} S_{0}+q \beta_{2} S_{0} \int_{0}^{h} e^{-\mu \tau} d \tau+(1-v) q \beta_{3} S_{0} \int_{h}^{\infty} e^{-\mu \tau} d \tau
$$

and

$$
V=\mathscr{V}_{2 \times 2}=\left[\begin{array}{cc}
\gamma+\eta+\mu & 0 \\
-\gamma & \alpha+\delta+\mu
\end{array}\right]
$$

Thus

$$
K V^{-1}=\left[\begin{array}{cc}
K V_{11}^{-1} & K V_{12}^{-1} \\
0 & 0
\end{array}\right]
$$


such that

$$
K V_{11}^{-1}=\frac{\gamma\left(\beta_{1} S_{0}+q \beta_{2} S_{0} \int_{0}^{h} e^{-\mu \tau} d \tau+(1-v) q \beta_{3} S_{0} \int_{h}^{\infty} e^{-\mu \tau} d \tau\right)}{(\gamma+\eta+\mu)(\alpha+\delta+\mu)}
$$

and

$$
K V_{12}^{-1}=\frac{\beta_{1} S_{0}+q \beta_{2} S_{0} \int_{0}^{h} e^{-\mu \tau} d \tau+(1-v) q \beta_{3} S_{0} \int_{h}^{\infty} e^{-\mu \tau} d \tau}{\alpha+\delta+\mu} .
$$

So the characteristic polynomial of $K V^{-1}$ appears with

$$
\lambda\left(\lambda-\frac{\gamma\left(\beta_{1} S_{0}+q \beta_{2} S_{0} \int_{0}^{h} e^{-\mu \tau} d \tau+(1-v) q \beta_{3} S_{0} \int_{h}^{\infty} e^{-\mu \tau} d \tau\right)}{(\gamma+\eta+\mu)(\alpha+\delta+\mu)}\right)=0 .
$$

Thus, the spectral radius of the next generation matrix is

$$
\rho\left(K V^{-1}\right)=\frac{\gamma\left(\beta_{1} S_{0}+q \beta_{2} S_{0} \int_{0}^{h} e^{-\mu \tau} d \tau+(1-v) q \beta_{3} S_{0} \int_{h}^{\infty} e^{-\mu \tau} d \tau\right)}{(\gamma+\eta+\mu)(\alpha+\delta+\mu)} .
$$

Taking into

$$
S_{0}=\frac{b}{q+\mu}, \quad \int_{0}^{h} e^{-\mu \tau} d \tau=\frac{1-e^{-\mu h}}{\mu}, \quad \int_{h}^{\infty} e^{-\mu \tau} d \tau=\lim _{k \rightarrow \infty} \int_{h}^{k} e^{-\mu \tau} d \tau=\frac{e^{-\mu h}}{\mu}
$$

account that, the basic reproduction number of the system (2.5) is calculated in the form of

$$
\begin{aligned}
\mathscr{R}_{0} & =\rho\left(K V^{-1}\right)=\frac{b \gamma\left(\beta_{1}+q \beta_{2} \frac{1-e^{-\mu h}}{\mu}+(1-v) q \beta_{3} \frac{e^{-\mu h}}{\mu}\right)}{(q+\mu)(\gamma+\eta+\mu)(\alpha+\delta+\mu)} \\
& =\frac{b \gamma\left(\mu \beta_{1}+q \beta_{2}\left(1-e^{-\mu h}\right)+(1-v) q \beta_{3} e^{-\mu h}\right)}{\mu(q+\mu)(\gamma+\eta+\mu)(\alpha+\delta+\mu)} .
\end{aligned}
$$

\subsubsection{Existence and Uniqueness of Endemic Equilibrium Point}

In subsection 2.1.2, we see that the system (2.5) always has a disease-free equilibrium point. Now, we investigate the existence and uniqueness of endemic equilibrium point. If the constant solution $P_{E}\left(S^{*}, E^{*}, I^{*}\right)$ is the endemic equilibrium of (2.5), the positive constants $S^{*}, E^{*}$ and $I^{*}$ should satisfy the algebraic equations

$$
\begin{aligned}
0 & =b-\beta_{1} S^{*} I^{*}-(q+\mu) S^{*}, \\
0 & =\beta_{1} S^{*} I^{*}+q \beta_{2} S^{*} I^{*} \int_{0}^{h} e^{-\mu \tau} d \tau+(1-v) q \beta_{3} S^{*} I^{*} \int_{h}^{\infty} e^{-\mu \tau} d \tau-(\gamma+\eta+\mu) E^{*}, \\
0 & =\gamma E^{*}-(\alpha+\delta+\mu) I^{*} .
\end{aligned}
$$

From second equation of this system, we have

$$
S^{*} I^{*}\left(\beta_{1}+q \beta_{2} \frac{1-e^{-\mu h}}{\mu}+(1-v) q \beta_{3} \frac{e^{-\mu h}}{\mu}\right)=(\gamma+\eta+\mu) E^{*}
$$

and then

$$
\frac{E^{*}}{I^{*}}=\frac{\left(\mu \beta_{1}+q \beta_{2}\left(1-e^{-\mu h}\right)+(1-v) q \beta_{3} e^{-\mu h}\right) S^{*}}{\mu(\gamma+\eta+\mu)} .
$$

On the other hand, from third equation of the system (2.7), we write

$$
\frac{E^{*}}{I^{*}}=\frac{\alpha+\delta+\mu}{\gamma}
$$

Considering (2.8) and (2.9), we get

$$
S^{*}=\frac{\mu(\gamma+\eta+\mu)(\alpha+\delta+\mu)}{\gamma\left(\mu \beta_{1}+q \beta_{2}\left(1-e^{-\mu h}\right)+(1-v) q \beta_{3} e^{-\mu h}\right)} .
$$


By the way, let us express the $S^{*}$ in terms of $\mathscr{R}_{0}$.

$$
S^{*}=\frac{b}{(q+\mu) \mathscr{R}_{0}}
$$

Also, from first equation of the system (2.7)

$$
\begin{aligned}
I^{*} & =\frac{b-(q+\mu) S^{*}}{\beta_{1} S^{*}} \\
& =\frac{(q+\mu)\left(\mathscr{R}_{0}-1\right)}{\beta_{1}}
\end{aligned}
$$

and taking into account (2.9) we obtain

$$
\begin{aligned}
E^{*} & =\frac{(\alpha+\delta+\mu) I^{*}}{\gamma} \\
& =\frac{(q+\mu)(\alpha+\delta+\mu)\left(\mathscr{R}_{0}-1\right)}{\gamma \beta_{1}} .
\end{aligned}
$$

Therefore, we say that the system (2.5) has a unique endemic equilibrium point

$$
\begin{aligned}
P_{E} & =\left(S^{*}, E^{*}, I^{*}\right) \\
& =\left(\frac{b}{(q+\mu) \mathscr{R}_{0}}, \frac{(q+\mu)(\alpha+\delta+\mu)\left(\mathscr{R}_{0}-1\right)}{\gamma \beta_{1}}, \frac{(q+\mu)\left(\mathscr{R}_{0}-1\right)}{\beta_{1}}\right)
\end{aligned}
$$

for $\mathscr{R}_{0}>1$.

\subsection{Local and Global Stabilities of Disease-free Equilibria}

In this part, local and global stability of disease-free equilibrium point $P_{D F}$ of the model (2.5) are discussed.

Theorem 2.2. The disease-free equilibrium point $P_{D F}$ is locally asymptotically stable in $\Upsilon$ for $\mathscr{R}_{0}<1$.

Proof. The Jacobian matrix at $P_{D F}=\left(S_{0}, E_{0}, I_{0}\right)$ of the system (2.5) is

$$
J\left(P_{D F}\right)=\left[\begin{array}{ccc}
-\beta_{1} I_{0}-(q+\mu) & 0 & -\beta_{1} S_{0} \\
J_{21}\left(P_{D F}\right) & -(\gamma+\eta+\mu) & J_{23}\left(P_{D F}\right) \\
0 & \gamma & -(\alpha+\delta+\mu)
\end{array}\right]
$$

where

$$
J_{21}\left(P_{D F}\right)=\beta_{1} I_{0}+q \beta_{2} I_{0} \int_{0}^{h} e^{-\mu \tau} d \tau+(1-v) q \beta_{3} I_{0} \int_{h}^{\infty} e^{-\mu \tau} d \tau
$$

and

$$
J_{23}\left(P_{D F}\right)=\beta_{1} S_{0}+q \beta_{2} S_{0} \int_{0}^{h} e^{-\mu \tau} d \tau+(1-v) q \beta_{3} S_{0} \int_{h}^{\infty} e^{-\mu \tau} d \tau
$$

For the point $\left(S_{0}, E_{0}, I_{0}\right)=\left(\frac{b}{q+\mu}, 0,0\right), J\left(P_{D F}\right)$ corresponds to the following form

$$
J\left(P_{D F}\right)=\left[\begin{array}{ccc}
-(q+\mu) & 0 & -\frac{b \beta_{1}}{q+\mu} \\
0 & -(\gamma+\eta+\mu) & L \\
0 & \gamma & -(\alpha+\delta+\mu)
\end{array}\right],
$$

where

$$
L=\frac{b\left(\mu \beta_{1}+q \beta_{2}\left(1-e^{-\mu h}\right)+(1-v) q \beta_{3} e^{-\mu h}\right)}{\mu(q+\mu)} .
$$

The characteristic equation for this Jacobian matrix is

$$
\begin{aligned}
\operatorname{det}\left(J\left(P_{D F}\right)-\lambda I_{3}\right) & =(-(q+\mu)-\lambda)\left|\begin{array}{cc}
-(\gamma+\eta+\mu)-\lambda & L \\
\gamma & -(\alpha+\delta+\mu)-\lambda
\end{array}\right| \\
& =0 .
\end{aligned}
$$


Hence

$$
(-(q+\mu)-\lambda)\left(\lambda^{2}+[(\gamma+\eta+\mu)+(\alpha+\delta+\mu)] \lambda+(\gamma+\eta+\mu)+(\alpha+\delta+\mu)-\gamma L\right)=0,
$$

and so the characteristic equation (2.10) always have the negative root $-(q+\mu)$. The other eigenvalues of this equation come from the equation

$$
\lambda^{2}+[(\gamma+\eta+\mu)+(\alpha+\delta+\mu)] \lambda+[(\gamma+\eta+\mu)+(\alpha+\delta+\mu)]\left(1-\mathscr{R}_{0}\right)=0 .
$$

For this quadratic equation,

$$
\lambda_{1} \lambda_{2}=[(\gamma+\eta+\mu)+(\alpha+\delta+\mu)]\left(1-\mathscr{R}_{0}\right)
$$

and

$$
\lambda_{1}+\lambda_{2}=-(\gamma+\eta+\mu)-(\alpha+\delta+\mu)<0 .
$$

If $\mathscr{R}_{0}<1$ then $\lambda_{1} \lambda_{2}>0$ and so two roots of Eq. (2.11) are also negative. If $\mathscr{R}_{0}=1$ we say that one of roots of Eq.(2.11) is zero. On the other hand, when $\mathscr{R}_{0}>1$ one of roots of Eq. (2.11) has positive real parts. So the disease-free equilibrium $P_{D F}$ is locally asymptotically stable for $\mathscr{R}_{0}<1$; is stable for $\mathscr{R}_{0}=1$, and is unstable for $\mathscr{R}_{0}>1$.

To prove that the disease-free equilibrium point $P_{D F}$ is globally asymptotically stable for $\mathscr{R}_{0}<1$, we use to the global attractivity of it. To see this property of the $P_{D F}$, we need the following lemma.

Lemma 2.3. [18] Assume that $f$ be a bounded, real-valued function defined on $[0, \infty)$ and be twice differentiable with bounded second derivative. Also, let us define notations $f_{\infty}$ and $f^{\infty}$ as

$$
f_{\infty}=\liminf _{t \rightarrow \infty} f(t), \quad f^{\infty}=\limsup _{t \rightarrow \infty} f(t),
$$

where

$$
\begin{aligned}
\inf f(t) & =\inf \{f(u): u \in[t, \infty), t>0\}, \\
\sup f(t) & =\sup \{f(u): u \in[t, \infty), t>0\} .
\end{aligned}
$$

Letting $k \rightarrow \infty, t_{k} \rightarrow \infty$ and $f\left(t_{k}\right)$ converges to $f_{\infty}$ and $f^{\infty}$, then $\lim _{k \rightarrow+\infty} f^{\prime}\left(t_{k}\right)=0$.

Theorem 2.4. The disease-free equilibrium point $P_{D F}$ is globally asymptotically stable in $\Upsilon$ for $\mathscr{R}_{0}<1$.

Proof. We investigate that $P_{D F}$ is globally attractive. From the first equation of the system (2.5) we write

$$
\frac{d S}{d t} \leq b-(q+\mu) S(t)
$$

Let us say $\frac{d X}{d t}=b-(q+\mu) X(t)$. Clearly, a solution of the equation $\frac{d X}{d t}=b-(q+\mu) X(t)$ is a supper solution of $S(t)$. Therefore $X(t) \geq S(t)$ for every $t \geq 0$. We immediately note that $X(t) \rightarrow \frac{b}{(q+\mu)}$ while $t \rightarrow \infty$. Then for a given $\varepsilon_{S}>0$, there exists a $t_{0}$ such that

$$
S(t) \leq X(t) \leq \frac{b}{q+\mu}+\varepsilon_{S}
$$

for $t \geq t_{0}$. So we say $S^{\infty} \leq \frac{b}{q+\mu}+\varepsilon_{S}$. In the event of $\varepsilon_{S} \rightarrow 0$, we write

$$
S^{\infty} \leq \frac{b}{q+\mu}
$$

From the third equation of the system (2.5) we obtain

$$
I(t) \rightarrow \frac{\gamma \lim _{t \rightarrow \infty} E(t)}{\alpha+\delta+\mu} \leq \frac{\gamma E^{\infty}}{\alpha+\delta+\mu},
$$

as $t \rightarrow \infty$. So we can say

$$
I^{\infty} \leq \frac{\gamma E^{\infty}}{\alpha+\delta+\mu}
$$

Taking into account the inequation (2.13), from the second equation of the system (2.5) we write

$$
\begin{aligned}
E(t) & \rightarrow \frac{\beta_{1} S(t) I(t)}{\gamma+\eta+\mu}+\frac{q \beta_{2} I(t) \int_{0}^{h} e^{-\mu \tau} S(t-\tau) d \tau}{\gamma+\eta+\mu}+\frac{(1-v) q \beta_{3} I(t) \int_{h}^{\infty} e^{-\mu \tau} S(t-\tau) d \tau}{\gamma+\eta+\mu} \\
& \leq\left(\frac{b \beta_{1}}{(q+\mu)(\gamma+\eta+\mu)}+\frac{b q \beta_{2}\left(1-e^{-\mu h}\right)}{\mu(q+\mu)(\gamma+\eta+\mu)}+\frac{b(1-v) q \beta_{3} e^{-\mu h}}{\mu(q+\mu)(\gamma+\eta+\mu)}\right) I^{\infty}
\end{aligned}
$$


as $t \rightarrow \infty$. So we get

$$
E^{\infty} \leq\left(\frac{b\left(\mu \beta_{1}+q \beta_{2}\left(1-e^{-\mu h}\right)+(1-v) q \beta_{3} e^{-\mu h}\right)}{\mu(q+\mu)(\gamma+\eta+\mu)}\right) I^{\infty} .
$$

Substituting the inequality (2.15) into (2.14), we obtain

$$
\begin{aligned}
I^{\infty} & \leq\left(\frac{b \gamma\left(\mu \beta_{1}+q \beta_{2}\left(1-e^{-\mu h}\right)+(1-v) q \beta_{3} e^{-\mu h}\right)}{\mu(q+\mu)(\gamma+\eta+\mu)(\alpha+\delta+\mu)}\right) I^{\infty} \\
& =\mathscr{R}_{0} I^{\infty} .
\end{aligned}
$$

Also, substituting (2.14) into the inequality (2.15), we attain

$$
\begin{aligned}
E^{\infty} & \leq\left(\frac{b \gamma\left(\mu \beta_{1}+q \beta_{2}\left(1-e^{-\mu h}\right)+(1-v) q \beta_{3} e^{-\mu h}\right)}{\mu(q+\mu)(\gamma+\eta+\mu)(\alpha+\delta+\mu)}\right) E^{\infty} \\
& =\mathscr{R}_{0} E^{\infty} .
\end{aligned}
$$

Hence if $\mathscr{R}_{0}<1$, we say that $I^{\infty} \leq 0$ and $E^{\infty} \leq 0$.

On the other hand, clearly $I_{\infty} \geq 0$ and $E_{\infty} \geq 0$. So $I_{\infty}=I^{\infty}=0$ and $E_{\infty}=E^{\infty}=0$. Therefore $(E(t), I(t)) \rightarrow(0,0)$ while $t \rightarrow \infty$. Now we show that

$$
\lim _{t \rightarrow \infty} S(t)=\frac{b}{q+\mu} .
$$

According to Lemma 2.3, we can determine the sequences $\left(r_{n}\right)$ and $\left(s_{n}\right)$ such that

$$
S\left(r_{n}\right) \rightarrow S_{\infty} \text { and } S\left(s_{n}\right) \rightarrow S^{\infty}
$$

while $\left(r_{n}\right) \rightarrow \infty$ and $\left(s_{n}\right) \rightarrow \infty$.

Again, in accordance with the same Lemma, we have

$$
S^{\prime}\left(r_{n}\right) \rightarrow 0 \text { and } S^{\prime}\left(s_{n}\right) \rightarrow 0
$$

From (2.12) and (2.16), we obtain

$$
b-(q+\mu) \liminf _{t \rightarrow \infty} S(t)=0
$$

and

$$
b-(q+\mu) \limsup _{t \rightarrow \infty} S(t)=0
$$

Therefore $\lim _{t \rightarrow \infty} S(t)=\frac{b}{q+\mu}$. Consequently disease-free equilibrium point $P_{D F}$ is global attractive for $\mathscr{R}_{0}<1$.

We have concluded that disease-free equilibrium point is locally asymptotically stable with previous theorem. Since $P_{D F}$ is both locally asymptotically stable and globally attractive, it is globally asymptotically stable in $\Upsilon$ for $\mathscr{R}_{0}<1$.

\section{Conclusion}

When it is carried out immunization with the vaccine, not only the vaccinated individual is protected against infectious disease, but also indirectly, it is prevented from infecting other individuals. Therefore, if the number of vaccinated individuals against the disease in the society is how much higher, the probability of the occurrence of that disease is lower at that rate. It is even possible to eliminate some diseases completely. For example, through successful vaccination programs, diseases such as smallpox, measles, polio have been completely eradicated or have been reduced to almost non-existent levels. This situation has increased the interest to models with vaccine in dynamical systems.

In this paper, it has been investigated the disease-free dynamics of a time delayed SVEIR epidemic model with a different perspective from the models in the literature. For the model it has been obtained the threshold quantity $\mathscr{R}_{0}$, called as the basic reproduction number. Next, as $\mathscr{R}_{0}<1$, it has been shown that disease-free equilibrium is locally asymptotically stable and is globally attractive, and as a result of this, is globally asymptotically stable. Vaccination always has a strong effect for disease control by decreasing the basic reproduction number. So, when $\mathscr{R}_{0}<1$,with effective, preventative and sustained vaccinations the disease can disappear ultimately.

\section{References}

[1] S. Çakan, Dynamic analysis of a mathematical model with health care capacity for COVID-19 pandemic, Chaos, Solitons and Fractals, 139, 110033 (2020), 1-8.

[2] P. Kumar, V.S. Ertürk, The analysis of a time delay fractional COVID-19 model via Caputo type fractional derivative, Math Meth Appl Sci., (2020), 1-14. https://doi.org/10.1002/mma.6935

[3] Z. Zhang, A. Zeb, S. Hussain, E. Alzahrani, Dynamics of COVID-19 mathematical model with stochastic perturbation, Adv. Differ. Equ., 451 (2020), 1-12. https://doi.org/10.1186/s13662-020-02909-1

[4] W.O. Kermack, A.G. McKendrick, A contributions to the mathematical theory of epidemics, Proc. Roy. Soc. A, 115 (1927), $700-721$.

[5] N.F. Tehrani, M.R. Razvan, S. Yasaman, Global Analysis of a delay SVEIR epidemiological model, Iran. J. Sci. Technol. Trans. A Sci., 37A4 (2013), 483-489.

[6] R. Xu, Global stability of a delayed epidemic model with latent period and vaccination strategy, Appl. Math. Model., 36 (2012), $5293-5300$. 
[7] A.A. Momoh, M.O. Ibrahim, B.A. Madu, Stability Analysis of an infectious disease free equilibrium of Hepatitis B model, Research Journal of Applied Sciences, Engineering and Technology, 3(9) (2011), 905-909.

[8] C. Wu, Z. Jiang, Global stability for the disease free equilibrium of a delayed model for malaria transmission, Int. Journal of Math. Analysis, 6(38) (2012), 1877-1881.

[9] C. Wu and Y. Zhang, Stability Analysis for the disease free equilibrium of a discrete malaria model with two delays, Intelligent Computing Theories and Applications. ICIC 2012, LNAI 7390 (2012), 341-349.

[10] R. Akbari, A.V. Kamyad, A.A. Heydari, A. Heydari, The analysis of a disease-free equilibrium of hepatitis B model, Sahand Communications in Mathematical Analysis (SCMA), 3(2) (2016), 1-11.

[11] J.C. Kamgang, G. Sallet, Computation of threshold conditions for epidemiological models and global stability of the disease-free equilibrium (DFE), Math. Biosci., 213 (2008), 1-12.

[12] J.C. Kamgang, G. Sallet, Global asymptotic stability for the disease free equilibrium for epidemiological models, Comptes Rendus Mathematique, 341(7) (2005), 433-438

[13] K.M. Bello, I.N. Akinwande, S. Abdurhman, A.F. Kuta, Y. Bello, Global stability analysis of the disease-free equilibrium state of a mathematical model of trypanosomiasis, J. Appl. Sci. Environ. Manage., 23(2) (2019), 201-208.

[14] K.B. Blyuss, Y.N. Kyrychko. Instability of disease-free equilibrium in a model of malaria with immune delay. Math Biosci. 248 (2014), 54-6. doi: 10.1016/j.mbs.2013.12.005. Epub 2013 Dec 25. PMID: 24373861.

[15] S. Lakshmikantham, S. Leela, A.A. Martynyuk, Stability Analysis of Nonlinear Systems, Marcel Dekker, Inc., New York, 1989.

[16] P.V.D. Driessche, J. Watmough, Reproduction numbers and sub-threshold endemic equilibria for compartmental models of disease transmission, Math.Biosci., 180 (2002), 29-48.

[17] O. Diekmann, J.A.P. Heesterbeek, J.A.J Metz, On the definition and the computation of the basic reproduction ratio $\mathscr{R}_{0}$ in models for infectious diseases in heterogeneous populations, Journal of Mathematical Biology, 28(4) (1990), 365-382.

[18] R.H. Thieme, Persitence under relaxed point dissipativity (with application to an endemic model), SIAM J. Math. Anal., 24 (1993), $407-435$. 